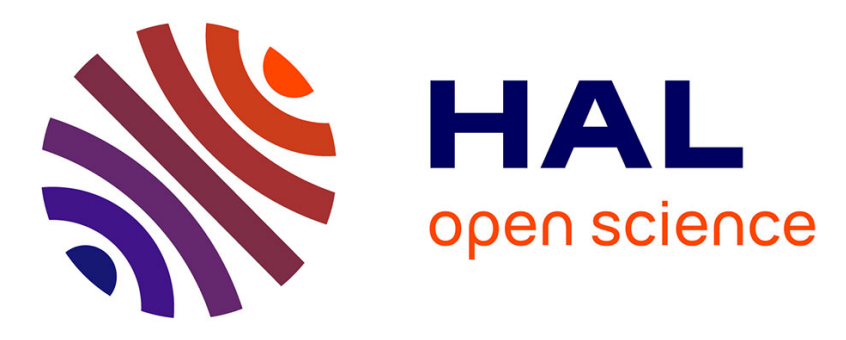

\title{
Just Noticeable Quantization Levels for High Dynamic Range Images
}

Sevim Begüm Sözer, Alper Koz, Ahmet Oguz Akyuz, Emin Zerman, Giuseppe Valenzise, Frédéric Dufaux

\section{To cite this version:}

Sevim Begüm Sözer, Alper Koz, Ahmet Oguz Akyuz, Emin Zerman, Giuseppe Valenzise, et al.. Just Noticeable Quantization Levels for High Dynamic Range Images. IEEE International Conference on Image Processing (ICIP'2020), Oct 2020, Abu Dhabi, United Arab Emirates. pp.1201-1205, 10.1109/ICIP40778.2020.9190931 . hal-02609440

\section{HAL Id: hal-02609440 \\ https://hal.science/hal-02609440}

Submitted on 13 Jun 2020

HAL is a multi-disciplinary open access archive for the deposit and dissemination of scientific research documents, whether they are published or not. The documents may come from teaching and research institutions in France or abroad, or from public or private research centers.
L'archive ouverte pluridisciplinaire HAL, est destinée au dépôt et à la diffusion de documents scientifiques de niveau recherche, publiés ou non, émanant des établissements d'enseignement et de recherche français ou étrangers, des laboratoires publics ou privés. 


\title{
JUST NOTICEABLE QUANTIZATION LEVELS FOR HIGH DYNAMIC RANGE IMAGES
}

\author{
Sevim Begüm Sözer ${ }^{a, b}$, Alper Koz ${ }^{a}$, Ahmet Oğuz Akyüz ${ }^{b}$, Emin Zerman ${ }^{c}$, Giuseppe Valenzise ${ }^{d}$, and \\ Frederic Dufaux ${ }^{d}$
}

\author{
${ }^{\mathrm{a} C}$ Center for Image Analysis (OGAM), ${ }^{\mathrm{b}}$ Department of Computer Engineering, \\ Middle East Technical University, Balgat, 06800, Ankara, Turkey \\ 'School of Computer Science and Statistics, Trinity College Dublin, Dublin, Ireland \\ ${ }^{d}$ Université Paris-Saclay, CNRS, CentraleSupelec, Laboratoire des Signaux et Systèmes \\ 91190, Gif-sur-Yvette, France
}

\begin{abstract}
Just noticeable quantization levels, which are conventionally used in picture coding, have been mainly developed for standard 8-bit images and low dynamic range (LDR) typical screens. The quantization levels however have not been adapted yet for high dynamic range (HDR) imaging and its accompanied HDR displays, which can reach up to a peak luminance of $4000 \mathrm{~cd} / \mathrm{m}^{2}$. This study proposes an experimental methodology on HDR displays to determine just noticeable quantization levels for discrete cosine transform (DCT) coefficients on high luminance images. In the first stage of the proposed method, the quantization noise patterns for different DCT frequencies at different mean luminances are rendered by predicting the LED and LCD values of the two layer HDR display. Then, a two alternative forced choice based psychovisual experimental procedure using geometric search and QUEST methodology is realized by randomly presenting the rendered quantization noise at different amplitudes to the subjects in order to determine the just noticeable levels. The experiments are performed over 3 subjects for 30 different frequencies of $8 \times 8$ DCT patterns at mean luminances of $100 \mathrm{~cd} / \mathrm{m}^{2}$ and $1000 \mathrm{~cd} / \mathrm{m}^{2}$. The results are interpreted with respect to frequency and luminance changes and from the point of utilized methodology, namely geometric search and QUEST.
\end{abstract}

Index Terms-just noticeable quantization levels, DCT, HDR, QUEST, image rendering

\section{INTRODUCTION}

High dynamic range (HDR) imaging [1] is proposed as a break through attempt in the last decades to provide more realistic and artistic views for digital imaging and computer graphics, and related cinema, television, and game industries. The main goal of this attempt is to capture, encode, transmit, and display the accurate physical luminance values in floating points for each pixel rather than 8-bit intensity values. The compression and transmission of such a floating point representation have been however one of the main obstacles for the proliferation of this technology to the consumer electronics market.

The compression of HDR content have been until now approached in two ways. The first approach was to provide backward compatibility to the existing 8-bit low dynamic range devices and displays [2-7]. The main stream in this class maps the floating point images or video frames into 8-bit and utilizes the standard codecs for encoding. After the 8-bit content is decoded, it can be directly viewed on an LDR display. Alternatively, the resulting LDR images are inversely mapped to the floating point in the case of an HDR display. The main focus of the methods in this category was mainly the design of the most suitable tone mapping (TM) curve for the 8-bit conversion. While some methods [2, 3] find the best TM curve from the point of rate distortion compensation, some others $[4,5]$ include also the quality of the generated LDR images into the formulation. The second approach [8-10] uses the high profile of existing codecs for the native encoding of floating point HDR images. Rather than mapping to 8bit images, the HDR content is first converted to 9-14 bits and encoded with the high profile of standard codecs. The images are then re-converted to floating points in the decoder side and viewed on an HDR display.

From the point of coding research, both approaches can be in fact regarded as a pragmatic engineering approach, which uses the available tools for standard coding without touching the core aspects of the HDR content. Different than its 8-bit counterparts, the coding of an HDR content formed of physical luminance values in floating points deserves a more fundamental research, which investigates the limitations and characteristics of human visual system (HVS) to be utilized for coding $[11,12]$. Following a similar path for the determination of just noticeable quantization levels for transform coefficients of LDR images [11], this paper proposes an experimental method to find those quantization levels for $8 \times 8$ discrete cosine transform (DCT) coefficients on high luminance images, which are generated on an HDR display.

More specifically, the proposed experimental method first renders the quantization noise patterns for different DCT frequencies at different amplitudes and mean luminances on an HDR screen by appropriately estimating the input values for the LED and LCD panels of the display. Then, the just noticeable quantization levels are determined for different frequencies and mean luminances by presenting the rendered patterns at different amplitudes to the subjects by a two alternative forced choice (2AFC) based psychovisual procedure using both geometric search and QUEST methodology [13]. The quantization levels are determined over 3 subjects for two mean luminance levels of 100 $\mathrm{cd} / \mathrm{m}^{2}$ and $1000 \mathrm{~cd} / \mathrm{m}^{2}$ and 30 DCT frequencies. The results are compared with respect to the changes in the mean luminance and frequencies, and the utilized psychovisual procedure, geometric search and QUEST.

The next section explains the main stages of the proposed experimental methodology. Section 3 gives and discusses the 
results of the pshychovisual tests. Finally, the conclusions are presented in the last section.

\section{PROPOSED EXPERIMENTAL METHODOLOGY}

The proposed experimental methodology is formed of three stages:

1) The HDR images for the quantization noise at different amplitudes for different DCT frequencies and mean luminances are constructed.

2) The constructed quantization noise patterns are rendered on an HDR display by properly determining the LED and LCD values.

3) The psychovisual experimental procedure based on geometric search and QUEST is applied to the subjects to determine the just noticeable quantization level for each 8x8 DCT frequency.

The following subsection provides the details of each stage.

\subsection{Construction of Quantization Noise Patterns for different} DCT frequencies and mean luminances

A procedure, similar to the case of LDR images [11], is adopted for the generation of quantization noise patterns for different frequencies by changing the mean luminance of the patterns accordingly for the HDR images:

$I_{m, n}(x, y)=I_{\text {avg }}+Q_{m, n} \cos \left(\frac{\pi m}{2 N}[2 x+1]\right) \cos \left(\frac{\pi n}{2 N}[2 y+1]\right)$

In the given equation, $I_{\text {avg }}$ refers to the mean luminance level of the image, $m$ and $n$ denotes the horizontal and vertical frequencies, and $Q_{m, n}$ represents the quantization residue after the quantization of the $(m, n)$ 'th DCT coefficient. The quantization noise at different levels (amplitudes) for the frequency $(m, n)$ is formed by changing the coefficient, $Q_{m, n}$ in the tests. The quantization noise pattern is placed to the center of an HD size HDR image, whose average value is fixed as $I_{\text {avg }}$, as illustrated in Figure 1 (a). Note that the quantization noise is illustrated for 9 blocks $(3 \times 3)$ of size $8 \times 8$ in the example and $Q_{m, n}$ is randomly drawn from a uniform distribution between $[-Q / 2, Q / 2]$, where $Q$ is the investigated quantization value.

\subsection{Rendering of the Constructed Quantization Noise Patterns on HDR Display}

As the first solution, the basic rendering algorithms in the literature $[14,15]$ are utilized to render the quantization noise pattern on the HDR display in a closest manner to the real physical values. However, a halo artifact is observed on the generated images on the HDR display due to the sharp changes in the middle of the screen, where the quantization noise is placed, as shown in Figure 1 (b). Accordingly, a new rendering algorithm is developed by considering the fact that the background is fixed for the generated patterns.

The proposed algorithm fixes all the LCD pixels values to 255 and then changes the LED values between 0 and 255. The mean luminance of the HDR display is measured with a luminance meter and recorded as a function of LED values. Figure 2 illustrates the resulting curve for the change of mean luminance values with respect to LED values. When all the LEDs on the display are utilized at the same time to get a uniform background, the overall mean luminance that can be obtained on the display is about 1500 $\mathrm{cd} / \mathrm{m}^{2}$ due to the power limitations. After the LED values of about 100 , the mean luminance is almost saturated on the display.

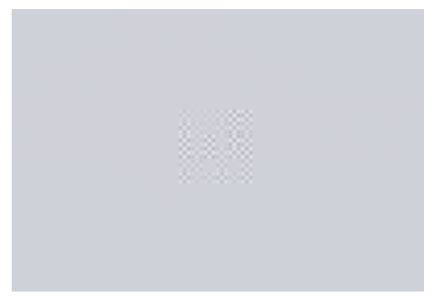

(a)

(b)

Fig. 1. (a) An example for the generated quantization noise pattern for a DCT frequency of $(3,3)$ (b) An example for the halo artifact in the center of the rendered view by using a baseline rendering algorithm [14].

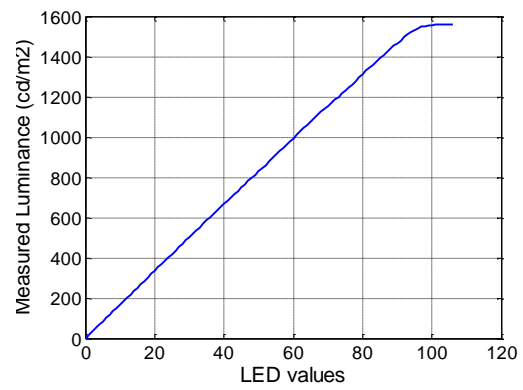

Fig. 2. The generated mean luminance levels for different LED values on the HDR display.

\begin{tabular}{|c|c|c|c|c|c|c|c|}
\hline $\mathrm{X}$ & $\mathrm{X}$ & $\mathrm{X}$ & $\mathrm{X}$ & & $\mathrm{X}$ & & $\mathrm{X}$ \\
\hline $\mathrm{X}$ & $\mathrm{X}$ & $\mathrm{X}$ & $\mathrm{X}$ & & $\mathrm{X}$ & & $\mathrm{X}$ \\
\hline $\mathrm{X}$ & $\mathrm{X}$ & $\mathrm{X}$ & & & & & \\
\hline $\mathrm{X}$ & $\mathrm{X}$ & & $\mathrm{X}$ & & $\mathrm{X}$ & & $\mathrm{X}$ \\
\hline & & & & & & & \\
\hline $\mathrm{X}$ & $\mathrm{X}$ & & $\mathrm{X}$ & & $\mathrm{X}$ & & $\mathrm{X}$ \\
\hline & & & & & & & \\
\hline $\mathrm{X}$ & $\mathrm{X}$ & & $\mathrm{X}$ & & $\mathrm{X}$ & & $\mathrm{X}$ \\
\hline
\end{tabular}

Fig. 3. The selected 30 DCT frequencies for the psychovisual tests

The proposed rendering algorithm using the obtained curve is as follows:

1) The HDR image and its mean luminace are inputted to the algorithm. Given the mean luminance, the corresponding closest LED value is found on the curve and this value is written to all the LED values using the DVI+ mode of the utilized SIM2 HDR Display.

2) The LCD values are calculated by using the HDR image and the LED values,

$$
L C D_{k}=\left(\frac{I_{k}}{L E D_{k} * P S F}\right)^{\delta_{k}}
$$

where $I_{k}$ for $k=1,2,3$ corresponds to $\mathrm{R}, \mathrm{G}, \mathrm{B}$ color channels respectively, $\delta_{k}$ is the gamma scaling factor for each channel, and PSF refers to the point spread function of the LEDs. The patterns are formed only in gray color by taking the $R, G, B$ values equal.

\subsection{Proposed Procedure for Psychovisual Tests}

The quantization noise patterns are formed for 30 different $8 \times 8$ DCT frequencies, illustrated in Figure 3, at two luminance levels 


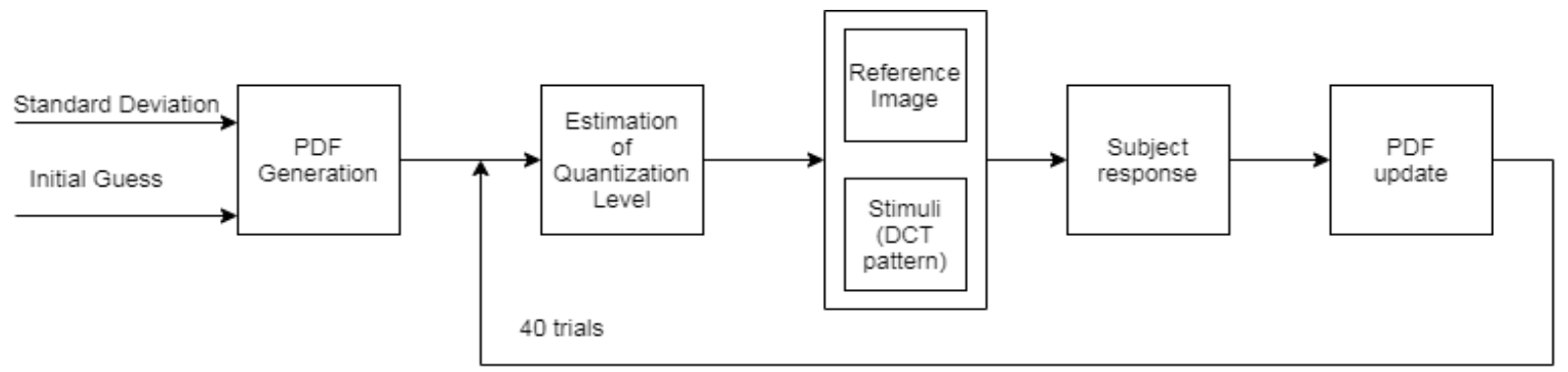

Fig. 4. The QUEST procedure to determine the just noticeable quantization levels for DCT frequencies on high luminance images

of $100 \mathrm{~cd} / \mathrm{m}^{2}$ and $1000 \mathrm{~cd} / \mathrm{m}^{2}$ for different quantization values. Then, these patterns are presented to the subjects with a two forced choice alternative choice (2AFC) procedure to find the just noticeable quantization levels.

The proposed 2AFC psychovisual test is formed of two phases, which combines geometric search and QUEST. In the first phase, the quantization levels are obtained for 3 subjects with a geometric search for each DCT frequency and then, the mean and standard deviation of the quantization levels for the geometric search are utilized as inputs in order to provide a more accurate initial distribution for QUEST in the second phase.

The geometric search procedure involves the following technical steps:

1) Beginning from the DCT quantization noise with the highest amplitude, the quantization noise pattern (stimulus) and a gray level HDR image with the same mean luminance (reference) are displayed to the subject in a random order for 0.5 seconds with an interval of 0.25 seconds.

2) The subject is asked if any difference is observed between two images. The subject presses ' $y$ ' from the keyboard, if any difference is observed. Otherwise, ' $n$ ' is selected. The next trial does not begin until a response is given.

3) If the answer is yes/no, then the amplitude of the quantization noise for the next trial is decreased/increased. The amount of change in the amplitude of the quantization noise in each iteration is calculated as the half of the difference between the amplitude values in the latest two trials.

4) When this difference is lower than a certain threshold, then the experiment is terminated.

The amplitude of the quantization noise pattern in the latest trial is considered as that subject's just noticeable quantization level for that frequency. Then, the average and standard deviation of the quantization levels for 3 subjects are inputted to the QUEST procedure [13]. QUEST is a statistical psychovisual procedure, which iteratively updates the estimate of the quantization level with respect to its distribution obtained from the history of the given answers until that iteration. The procedure involves the following stages, as illustrated in Figure 4:

1) The initial probability density function (PDF) for the quantization level is formed with the mean and standard deviation obtained from the geometric search.
2) The amplitude of the quantization noise pattern is drawn from the distribution. The stimulus pattern, formed with the given amplitude, and the reference pattern are presented to the subject in a random order for 0.5 seconds with an interval of 0.25 seconds.

3) The subject is asked in which period of time he sees the stimulus. The response is saved as correct or wrong and a feedback is given to the subject with a beep sound for the wrong answer.

4) The PDF is updated with the answer of the subject.

After forty trials, the experiment is terminated. A Weibull function is fitted to the QUEST results and the quantization level corresponding to the confidence of 0.82 is assumed as the just noticeable quantization level for that frequency [11].

\section{EXPERIMENTAL RESULTS}

The experiments are performed on 3 subjects between 25 and 30 years old without any vision defect in a dark room without window and external light sources. The subjects are located to a distance of about 1.85 meter from the display, which corresponds to a 30 degree of viewing angle with a 64 pixels/degree. The interface for the pyschovisual tests is prepared in Matlab environment by using Psychtoolbox [16].

Figure 5 (a) indicates an example for the change of the amplitude of the quantization noise patterns with respect to iterations during the experiments for the 8x8 DCT frequencies of $(1,1)$ and $(7,1)$ at a mean luminance of $1000 \mathrm{~cd} / \mathrm{m}^{2}$ for the geometric search for Subject 1 (S1). The same graphs are also presented in Figure 5 (b) for QUEST. In both cases, the amplitude of the pattern are converging to a level with the iterations. While QUEST indicates a slower convergence rate with a longer number of iterations, the number of iterations for geometric search is just 9 , as the difference in the amplitudes of the consecutive trials gets smaller than the threshold. The convergence level is higher for the frequency of $(7,1)$ compared to the frequency of $(1,1)$ in accordance with the lower sensitivity of the HVS to the higher spatial frequencies.

Figure 5 (c) and (d) illustrates the quantization levels with respect to the vertical frequency, when the horizontal frequency is fixed to 7 for the 3 subjects, for geometric search and QUEST, respectively. The quantization levels have similar characteristics for all the subjects for increasing spatial frequencies for the geometric search. The same behavior is also observed for the increasing horizontal frequencies as well. The quantization levels have almost geometric increase for increasing spatial frequencies. A similar characteristic is also observed for Subject 2 and 3, for the QUEST tests. However, there is a distinctive outlier for the 


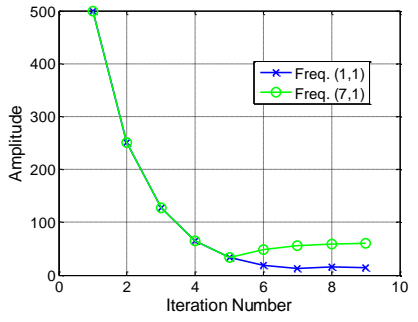

(a)

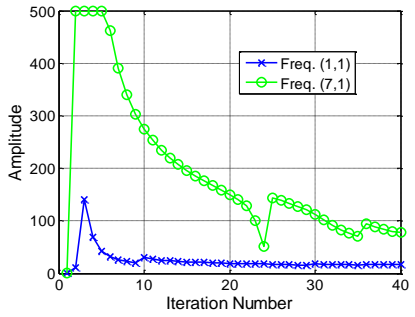

(b)

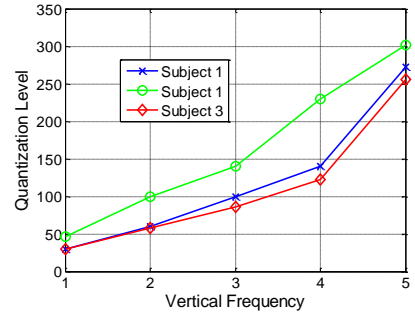

(c)

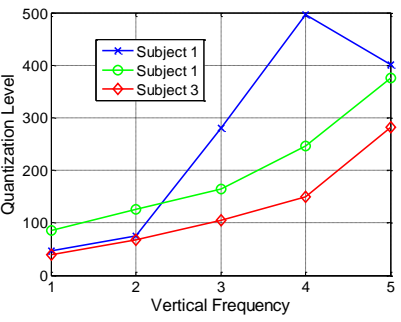

(d)

Fig. 5. (a) The change in the amplitudes of the quantization patterns with respect to iteration number for Geometric Search and (b) for QUEST. (c) Just noticeable quantization levels as a function of vertical frequency for all subjects for Geometric Search, and (d) for QUEST

frequency of $(7,4)$ for Subject 1. Such behavior for some frequencies in QUEST experiments can be linked with the high number of iterations and long duration of the tests for 30 DCT frequencies, which might make the subjects lose their attention. These outliers are filtered by taking the median of the resulting just noticeable quantization levels for the three subjects.

As another aspect of the comparisons, Figure 6 gives the quantization levels as the median of 3 subjects for different methodologies and mean luminances. The quantization levels for the QUEST are higher compared to the geometric search for both luminance levels of $100 \mathrm{~cd} / \mathrm{m}^{2}$ and $1000 \mathrm{~cd} / \mathrm{m}^{2}$. Noting that the QUEST further refine the results obtained with the geometric search, this refinement indicate itself as an increase in the quantization levels with a confidence level of 0.82 . The quantization levels for $1000 \mathrm{~cd} / \mathrm{m}^{2}$ is significantly higher than the case of $100 \mathrm{~cd} / \mathrm{m}^{2}$, as expected.

Figure 7 gives the ultimate quantization tables obtained with the geometric search and QUEST for all the 30 DCT frequencies and mean luminances of $100 \mathrm{~cd} / \mathrm{m}^{2}$ and $1000 \mathrm{~cd} / \mathrm{m}^{2}$. As mentioned in the previous specific cases, the increase in the quantization levels for higher luminance and frequencies are quite observable in the tables, while QUEST provides higher quantization levels compared to the geometric search. The characterization of this increase with respect to the frequency and luminance changes and its modelling will be further investigated in the future research.

\section{CONCLUSIONS}

Owing to the recent advances to generate HDR images in new HDR displays, an experimental psyhovisual method is proposed to determine the just noticeable quantization levels for DCT patterns with high mean luminances. The experiments are performed for 30 different DCT frequencies with mean luminances of $100 \mathrm{~cd} / \mathrm{m}^{2}$ and $1000 \mathrm{~cd} / \mathrm{m}^{2}$. In accordance with the basic characteristics of HVS, the quantization levels are observed to be higher for higher lumimances and spatial frequencies. The future work will extend the experiments for different mean luminances and model the quantization levels as a function of the mean luminances and spatial frequencies. The ultimate goal would be to integrate the developed model for native compression of HDR content.

\section{ACKNOWLEDGMENT}

This research is supported by The Scientific and Technical Research Council of Turkey (TÜBİTAK) and the French Ministry of Foreign Affairs under the common programme BOSPHORUS with the project number $115 \mathrm{E} 830$.

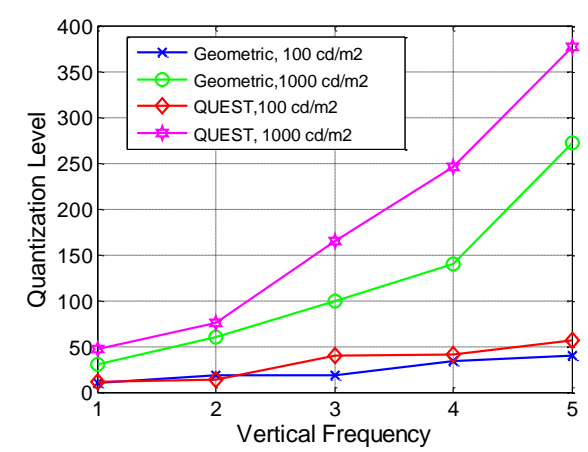

Fig. 6. The median of the just noticeable quantization levels for three subjects as a function of vertical frequency for different mean luminances and different methods

\begin{tabular}{|llllllll|}
\hline 4 & 4 & 4 & 4 & $\mathrm{X}$ & 4 & $\mathrm{X}$ & 8 \\
4 & 4 & 4 & 4 & $\mathrm{X}$ & 6 & $\mathrm{X}$ & 14 \\
4 & 4 & 4 & $\mathrm{X}$ & $\mathrm{X}$ & $\mathrm{X}$ & $\mathrm{X}$ & $\mathrm{X}$ \\
4 & 4 & $\mathrm{X}$ & 4 & $\mathrm{X}$ & 10 & $\mathrm{X}$ & 20 \\
$\mathrm{X}$ & $\mathrm{X}$ & $\mathrm{X}$ & $\mathrm{X}$ & $\mathrm{X}$ & $\mathrm{X}$ & $\mathrm{X}$ & $\mathrm{X}$ \\
4 & 6 & $\mathrm{X}$ & 8 & $\mathrm{X}$ & 16 & $\mathrm{X}$ & 30 \\
$\mathrm{X}$ & $\mathrm{X}$ & $\mathrm{X}$ & $\mathrm{X}$ & $\mathrm{X}$ & $\mathrm{X}$ & $\mathrm{X}$ & $\mathrm{X}$ \\
10 & 18 & $\mathrm{X}$ & 18 & $\mathrm{X}$ & 34 & $\mathrm{X}$ & 40 \\
\hline
\end{tabular}

(a)

\begin{tabular}{|llllllll|}
\hline 2 & 1 & 2 & 2 & $\mathrm{X}$ & 4 & $\mathrm{X}$ & 16 \\
2 & 3 & 3 & 4 & $\mathrm{X}$ & 9 & $\mathrm{X}$ & 14 \\
3 & 3 & 4 & $\mathrm{X}$ & $\mathrm{X}$ & $\mathrm{X}$ & $\mathrm{X}$ & $\mathrm{X}$ \\
2 & 3 & $\mathrm{X}$ & 7 & $\mathrm{X}$ & 12 & $\mathrm{X}$ & 18 \\
$\mathrm{X}$ & $\mathrm{X}$ & $\mathrm{X}$ & $\mathrm{X}$ & $\mathrm{X}$ & $\mathrm{X}$ & $\mathrm{X}$ & $\mathrm{X}$ \\
4 & 11 & $\mathrm{X}$ & 11 & $\mathrm{X}$ & 29 & $\mathrm{X}$ & 40 \\
0 & $\mathrm{X}$ & $\mathrm{X}$ & $\mathrm{X}$ & $\mathrm{X}$ & $\mathrm{X}$ & $\mathrm{X}$ & $\mathrm{X}$ \\
11 & 14 & $\mathrm{X}$ & 40 & $\mathrm{X}$ & 41 & $\mathrm{X}$ & 57 \\
\hline
\end{tabular}

(c)

\begin{tabular}{|llllllll|}
\hline 8 & 8 & 8 & 8 & $\mathrm{X}$ & 14 & $\mathrm{X}$ & 24 \\
8 & 14 & 16 & 28 & $\mathrm{X}$ & 28 & $\mathrm{X}$ & 60 \\
8 & 14 & 10 & $\mathrm{X}$ & $\mathrm{X}$ & $\mathrm{X}$ & $\mathrm{X}$ & $\mathrm{X}$ \\
8 & 16 & $\mathrm{X}$ & 24 & $\mathrm{X}$ & 46 & $\mathrm{X}$ & 92 \\
$\mathrm{X}$ & $\mathrm{X}$ & $\mathrm{X}$ & $\mathrm{X}$ & $\mathrm{X}$ & $\mathrm{X}$ & $\mathrm{X}$ & $\mathrm{X}$ \\
16 & 28 & $\mathrm{X}$ & 40 & $\mathrm{X}$ & 60 & $\mathrm{X}$ & 176 \\
$\mathrm{X}$ & $\mathrm{X}$ & $\mathrm{X}$ & $\mathrm{X}$ & $\mathrm{X}$ & $\mathrm{X}$ & $\mathrm{X}$ & $\mathrm{X}$ \\
30 & 60 & $\mathrm{X}$ & 100 & $\mathrm{X}$ & 140 & $\mathrm{X}$ & 272 \\
\hline
\end{tabular}

(b)

\begin{tabular}{|llllllll|}
\hline 13 & 13 & 14 & 12 & $\mathrm{X}$ & 24 & $\mathrm{X}$ & 57 \\
27 & 23 & 21 & 22 & $\mathrm{X}$ & 38 & $\mathrm{X}$ & 71 \\
12 & 22 & 28 & $\mathrm{X}$ & $\mathrm{X}$ & $\mathrm{X}$ & $\mathrm{X}$ & $\mathrm{X}$ \\
11 & 25 & $\mathrm{X}$ & 49 & $\mathrm{X}$ & 87 & $\mathrm{X}$ & 172 \\
$\mathrm{X}$ & $\mathrm{X}$ & $\mathrm{X}$ & $\mathrm{X}$ & $\mathrm{X}$ & $\mathrm{X}$ & $\mathrm{X}$ & $\mathrm{X}$ \\
18 & 50 & $\mathrm{X}$ & 62 & $\mathrm{X}$ & 161 & $\mathrm{X}$ & 232 \\
$\mathrm{X}$ & $\mathrm{X}$ & $\mathrm{X}$ & $\mathrm{X}$ & $\mathrm{X}$ & $\mathrm{X}$ & $\mathrm{X}$ & $\mathrm{X}$ \\
47 & 75 & $\mathrm{X}$ & 165 & $\mathrm{X}$ & 246 & $\mathrm{X}$ & 376 \\
\hline
\end{tabular}

(d)
Fig. 7. The resulting just noticeable quantization tables (a) for geometric search, $100 \mathrm{~cd} / \mathrm{m}^{2}$ (b) for geometric search, $1000 \mathrm{~cd} / \mathrm{m}^{2}$ (c) for QUEST $100 \mathrm{~cd} / \mathrm{m}^{2}$, and (d) for QUEST, $1000 \mathrm{~cd} / \mathrm{m}^{2}$

\section{REFERENCES}

[1] Advanced High Dynamic Range Imaging by Francesco Banterle, Alessandro Artusi, Kurt Debattista, and Alan Chalmers, 278 pages by AK Peters (now CRC Press), 2011.

[2] Z. Mai, H. Mansour, R. Mantiuk, P. Nasiopoulos, R. Ward, and W. Heidrich, "Optimizing a Tone Curve for Backward-Compatible High 
Dynamic Range Image and Video Compression”, IEEE Transactions on Image Processing, pp. 1558-1571, Vol. 20, No. 6, June 2011.

[3] D. Gommelet, A. Roumy, C. Guillemot, M. Ropert and J. LeTanou, "Rate-Distortion Optimization of a Tone Mapping with SDR Quality Constraint for Backward-Compatible High Dynamic Range Compression", in Proc. IEEE International Conference on Image Processing (ICIP2016), Arizona, USA.

[4] A. Koz and F. Dufaux, "Methods for Improving the Tone Mapping for Backward Compatible High Dynamic Range Image and Video Compression", Elsevier Signal Processing: Image Communication, vol. 29, no. 2, pp. 274-292, February 2014.

[5] D. Gommelet, A. Roumy, C. Guillemot, M. Ropert, J. Le Tanou, "Gradient-based tone mapping for rate-distortion optimized backward-compatible high dynamic range compression", IEEE Transactions on Image Processing, vol. 26,no. 12, pp. 5936-5949, Dec. 2017.

[6] R. Boitard, R. Cozot, D. Thoreau, K. Bouatouch, "Motion-Guided Quantization for Video Tone Mapping”, in Proc. IEEE International Conference on Multimedia and Expo (ICME2014), Chengdu, China.

[7] R. Mantiuk, A. Efremov, K. Myszkowski, and H. P. Seidel, "Backward Compatible High Dynamic Range Mpeg Video Compression," ACM Trans. Graphics (Proc. SIGGRAPH), Vol. 25, No. 3, pp. 713-723, 2006.

[8] A. Motra and H. Thoma, "An adaptive logluv transform for high dynamic range video compression", in Proceedings of IEEE,ICIP, pp. 2061-2064,2010.
[9] J.-U. Garbas and H. Tahoma, "Temporally coherent luminance-toluma mapping for high dynamic range video coding with H.264/ AVC", in Proceedings of IEEE, ICASSP, pp.829-832, 2011.

[10] Y. Zhang, E. Reinhard and, D. Bull, "Perception-based high dynamic range video compression with optimal bit depth transformation", in Proceedings of IEEE, ICIP, pp.1321-1324, 2011.

[11] H. A. Peterson, A. J. Ahumada and A. B. Watson, "Visibility of DCT Quantization Noise", in Digest of Technical Papers, Society for Information Display, Playa, 1993,pp. 942-945.

[12] A. B. Watson, "DCT quantization matrices visually optimized for individual images", Proc. SPIE Human Vision Visual Processing and Digital Display IV, vol. 1913, pp. 202-216, 1993-Sep.

[13] A. B. Watson and D. G. Pelli, "Quest: a bayesian adaptive psychometric method.," Perception and Psycho-physics, 1983.

[14] E. Zerman, G. Valenzise, F. Dufaux, "A dual modulation algorithm for accurate reproduction of high dynamic range video" in proc. of IEEE 12th Image, Video and Multidimensional Signal Processing Workshop (IVMSP), 2016

[15] H. Seetzen, W. Heidrich, W. Stuerzlinger, G. Ward, L. Whitehead, M. Trentacoste, A. Ghosh, and A. Vorozcovs, "High dynamic range display systems," ACM Trans. Graph., vol. 23, no. 3, pp. 760-768, Aug. 2004.

[16] Psychtoolbox 3, [Online]. Available:http://psychtoolbox.org/ 